\title{
ОБОБЩЕННЫЕ ХАРАКТЕРИСТИКИ ЭНЕРГЕТИЧЕСКОГО СГЛАЖИВАЮЩЕГО Г-ОБРАЗНОГО ФИЛЬТРА
}

\author{
(Представил Н. Эпик)
}

Теория электрических фильтров достаточно хорошо разработана с учетом особенностей и характерных режимов работы маломощных (сигнальных) фильтров. Хотя эта теория верна и для мощных (энергетических), например сглаживающих фильтров, использование ее для решения инженерных задач в этой области не всегда удобно и наглядно. Если, например, при проектировании сигнальных фильтров обычно стараются их нагружать характеристическим сопротивлением, а также максимально использовать мощность источника сигнала, т. е. согласовать с источником питания и нагрузки, то энергетические сглаживающие фильтры обычно работают в области, очень далекой от этих оптимальных режимов. Известно, например, что максимальная мощность передается от источника энергии к нагрузке при равенстве внутренного сопротивления источника и сопротивления нагрузки. Но при этом энергетический КПД равен 0,5 , что явно не приемлемо для энергетических устройств. При КПД, равном 0,95 , величина внутреннего сопротивления источника питания составляет только $5,26 \%$ от сопротивления нагрузки.

Таким образом, можно выделить две основные отличительные особенности энергетических сглаживающих фильтров по сравнению с сигнальными фильтрами: ограничения по выбору КПД фильтра и работа, как правило, в несогласованном в смысле принятого для сигнальных фильтров режиме.

Для расчета и проектирования энергетических сглаживающих фильтров предложен ряд методик, входящих и в учебную литературу. Для Г-образного фильтра обычно по заданному коэффициенту сглаживания определяется произведение $L C$ и исходя из различных дополнительных условий (напр. получение индуктивной реакции фильтра, минимизации массы или габарита и т. п.) выбираются конкретные величины индуктивности дросселя и емкости конденсатора. При необходимости отдельно анализируется вопрос о подавлении или ограничении собственных резонансов, о перенапряжении и сверхтоках при переходных процессах и т. п. и принимаются дополнительные меры (дополнительные схемные элементы, специальные переключающие устройства и т. д.) для выполнения заданных требований по этим показателям. При этом потери в фильтре (т. е. КПД фильтра) учитываются обычно только при тепловом расчете его элементов.

В настоящей статье рассматриваются обобщенные характеристики энергетических сглаживающих Г-образных фильтров на базе минимального количества критериальных параметров, одним из которых является КПД фильтра, и приводятся вытекающие из этих характеристик 
зависимости для выбора элементов фильтра с учетом некоторых специфических требований, предъявляемых сглаживающим фильтрам.

Приводимые зависимости являются общими для данного типа фильтров и не относятся к какой-либо конкретной области их применения.

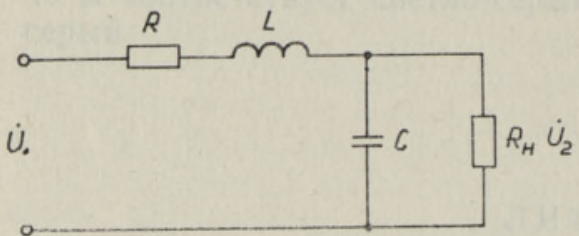

Рис. 1.

Рассмотрим $\Gamma$-образный сглаживающий $L C$-фильтр с чисто активной нагрузкой (рис. 1). Здесь $R-$ означает сопротивление дросселя. Для упрощения пренебрегаем влиянием внутренного сопротивления и индуктивности источника питания (или считаем их входящими в величины $L$ и $R$ ), т. е. считаем, что фильтр питается от источника напряжения. Также принимаем, что $R$ не зависит от частоты.

Коэффициент передачи напряжения такой цепи выражается

$$
K(j \omega)=\frac{\dot{U}_{2}}{\dot{U}_{1}}=\frac{1}{1+\frac{R}{R_{\mathrm{H}}}-\omega^{2} L C+j \omega\left(\frac{L}{R_{\mathrm{H}}}+R C\right) .} .
$$

Введя обозначения

$\Omega=\omega \sqrt{L C}-$ нормированная резонансная частота $L C$-контура,

$Q=\frac{\sqrt{L / C}}{R}-$ добротность $L C$-контура,

$\eta=\frac{1}{1+\frac{R}{R_{\text {H}}}}-$ КПД фильтра по постоянному току,

имеем

$$
K(j \Omega)=\frac{1}{\frac{1}{\eta}-\Omega^{2}+j \Omega\left[\frac{1}{Q}+Q\left(\frac{1}{\eta}-1\right)\right]} .
$$

Отсюда модуль коэффициента передачи напряжения

$$
K(\Omega)=\left\{\left(\frac{1}{\eta}-\Omega^{2}\right)^{2}+\Omega^{2}\left[\frac{1}{Q}+Q\left(\frac{1}{\eta}-1\right)\right]^{2}\right\}^{-1 / 2}
$$

и фаза

$$
\varphi=\operatorname{arctg} \frac{\Omega\left[\frac{1}{Q}+Q\left(\frac{1}{\eta}-1\right)\right]}{\frac{1}{\eta}-\Omega^{2}} .
$$

В дальнейшем рассмотрим коэффициент сглаживания фильтра, равный обратной величине коэффициента передачи напряжения. Его модуль

$$
k_{\mathrm{c}}=\frac{1}{K(\Omega)}=\sqrt{\left(\frac{1}{\eta}-\Omega^{2}\right)^{2}+\Omega^{2}\left[\frac{1}{Q}+Q\left(\frac{1}{\eta}-1\right)\right]^{2}} .
$$


Следует отметить, что в коэффициент сглаживания входит и не зависящее от частоты уменьшение выходного напряжения в результате деления на активные сопротивления $R$ и $R_{\text {н. }}$ Поэтому из $(5)$ следует, что при $\Omega=0$

$$
k_{c}=\frac{1}{\eta}-1
$$

и зависит от величины КПД

Из (4) и (5) следует, что при заданной постоянной величине $\eta$ частотные характеристики рассматриваемого фильтра зависят только от добротности $Q$ и определяются суммой в квадратных скобках. В этой сумме первый член убывает, а второй - возрастает при увеличении $Q$. Поэтому, как было указано в $\left[{ }^{1}\right]$, при $\eta=$ const эта сумма имеет одинаковую величину при двух различных значениях $Q=Q_{1}$ и $Q=Q_{2}$, которые связаны между собой выражением

$$
Q_{1} Q_{2}=\frac{1}{\frac{1}{\eta}-1}
$$

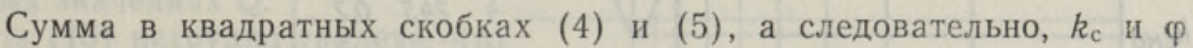
имеют при заданных $\eta$ и $\Omega$ минимальную величину, когда

$$
\frac{1}{Q}=Q\left(\frac{1}{\eta}-1\right)
$$

или

$$
Q_{1}=Q_{2}=Q_{\mathrm{M}}=\left(\frac{1}{\eta}-1\right)^{-1 / 2}
$$

На рис. 2 приведены для примера некоторые частотные зависимости коэффициента сглаживания $k_{\mathrm{c}}$ при $\eta=0,98$ и при различных значениях $Q$.

Для сглаживающего фильтра существенное значение имеет ход коэффициента сглаживания вблизи резонансной частоты $\Omega \approx 1$, где может иметь место нежелательное усиление входного сигнала, когда коэффициент сглаживания станет меньше единицы. Для предотвращения или подавления этого явления обычно рекомендуется ввести в фильтр элемент с большим затуханием, в основном в виде дополнительной $R C$-цепочки параллельно с основной емкостью $C\left[{ }^{2,3}\right]$. В последующем анализе, развивая подход приведенный в $\left[{ }^{4}\right]$, выводятся общие критерии, выполнение которых при выборе элементов фильтра позволяет подавлять резонансные явления без введения дополнительных схемных элементов.

Подставляя $Q_{\mathrm{M}}$ из $(7)$ в $(5)$, имеем после преобразования

$$
k_{\mathrm{C}=Q_{\mathrm{M}}}=\sqrt{\left(\Omega^{2}+\frac{1}{\eta}\right)^{2}-4 \Omega^{2}} .
$$

Последняя зависимость определяет наиболее низко расположенную частотную характеристику фильтра, т. е. минимально возможное значение коэффициента сглаживания при заданных $\eta$ и $\Omega$. На рис. 2 этому условию соответствует кривая 1 .

Из (5) следует, что в случае идеального резонанса, когда $\eta=1$ и $Q=\infty$, на частоте $\Omega=1 k_{\mathrm{c}}=0$, т. е. имеет место бесконечное усиление

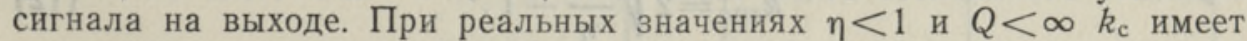
минимальную величину $k_{\mathrm{cm}}>0$ на частоте $\Omega_{\mathrm{M}}<1$ (напр., кривая 2 на рис. 2). 


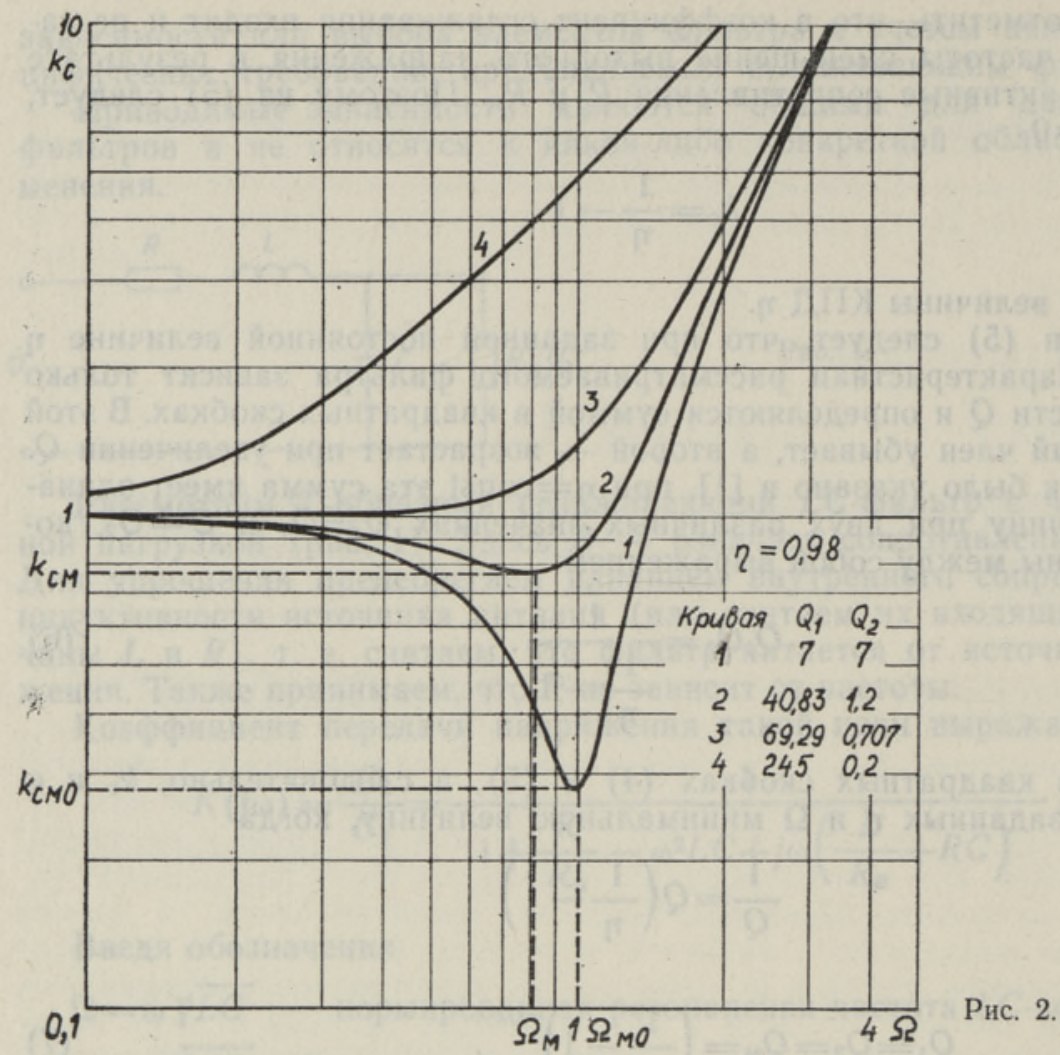

Для определения $k_{\mathrm{cm}}$ в точке минимума, найдем из (5) соответствующую этому величину $\Omega_{\mathrm{M}}$. Приравняв производную $k_{c}$ по $\Omega$ к нулю, найдем

$$
\Omega_{\mathrm{M}}=\sqrt{\frac{1}{\eta}-\frac{1}{2}\left[\frac{1}{Q}+Q\left(\frac{1}{\eta}-1\right)\right]^{2}} .
$$

Величину $k_{\mathrm{c}}$ в точке минимума найдем, подставляя $\Omega_{\mathrm{M}}$ из $(9)$ в (5). Проводя несложные преобразования, имеем

$$
k_{\mathrm{cM}}=\left[\frac{1}{Q}+Q\left(\frac{1}{\eta}-1\right)\right] \sqrt{\frac{1}{\eta}-\frac{1}{4}\left[\frac{1}{Q}+Q\left(\frac{1}{\eta}-1\right)\right]^{2}} .
$$

Забегая вперед, следует отметить, что последнее выражение имеет физический смысл только при значениях добротности $Q_{\mathrm{kp1}}>Q>Q_{\mathrm{kp2}}$, где $Q_{\mathrm{kp1}}$ и $Q_{\mathrm{kp2}}$ определены из (13).

Подставляя $Q_{\mathrm{M}}$ из $(7)$ в (9), найдем, что при данном $\eta$ наименьшее возможное значение $k_{\mathrm{c}}$ имеет место при

$$
\Omega_{\mathrm{MO}}=\sqrt{2-\frac{1}{\eta}} .
$$

Из (11) и (8) следует, что в этом случае и $k_{\text {см0 }}$ определяется только величиной $\eta$ :

$$
k_{\mathrm{cMO}}=2 \sqrt{\frac{1}{\eta}-1} .
$$

Из последнего выражения видно, что $k_{\text {см0 }} \geqslant 1$, если $\eta \leqslant 0,8$, т. е. эф- 
фект усиления не может иметь места при любых величинах добротности $Q$ и частоты $\Omega$, если КПД фильтра ниже 0,8 .

Для выполнения условия $k_{\mathrm{cm}} \geqslant 1$ при значениях $\eta>0,8$ необходимо соответственным образом выбрать величину $Q$. Подставляя $\Omega_{\mathrm{M}}$ из (9) в (5) и приравняв последнюю к единице, найдем критические величины $Q_{\mathrm{kp1}, 2}$

$$
Q_{\mathrm{kp} 1,2}=\left(\frac{\eta}{2}\right)^{1 / 2} \frac{\left[1-\left(1-\eta^{2}\right)^{1 / 2}\right]^{1 / 2} \pm\left[2 \eta-1-\left(1-\eta^{2}\right)^{1 / 2}\right]^{1 / 2}}{1-\eta} .
$$

Таким образом, условие $k_{\mathrm{cm}} \geqslant 1$ выполняется при двух значениях $Q \geqslant Q_{\mathrm{kp1}}$ или $Q \leqslant Q_{\mathrm{kp} 2}$, причем

$$
Q_{\mathrm{kp} 1} Q_{\mathrm{kp} 2}=\frac{1}{\frac{1}{\eta}-1},
$$

как следует и из (6). Зависимость $Q_{\mathrm{kp}}$ от $\eta$ приведена на рис. 3 (сплошная кривая). При $Q_{\mathrm{kp1}}>Q>Q_{\mathrm{kp} 2} k_{\mathrm{cm}}<1$ и имеет место усиление входного сигнала вблизи резонансной частоты, т. е. $U_{2 \mathrm{M}}>U_{1 \mathrm{M}}$. Из рис. 3 видно, что при $\eta \leqslant 0,8$ усиление отсутствует, т. е. $k_{c \mathrm{M}} \geqslant 1$ при любых значениях $Q$.

Из (13) нетрудно увидеть, что

$$
\lim _{\eta \rightarrow 1} Q_{\mathrm{kp1}}=\infty \quad \text { и } \quad \lim _{\eta \rightarrow 1} Q_{\mathrm{kp} 2}=\frac{1}{\sqrt{2}} .
$$

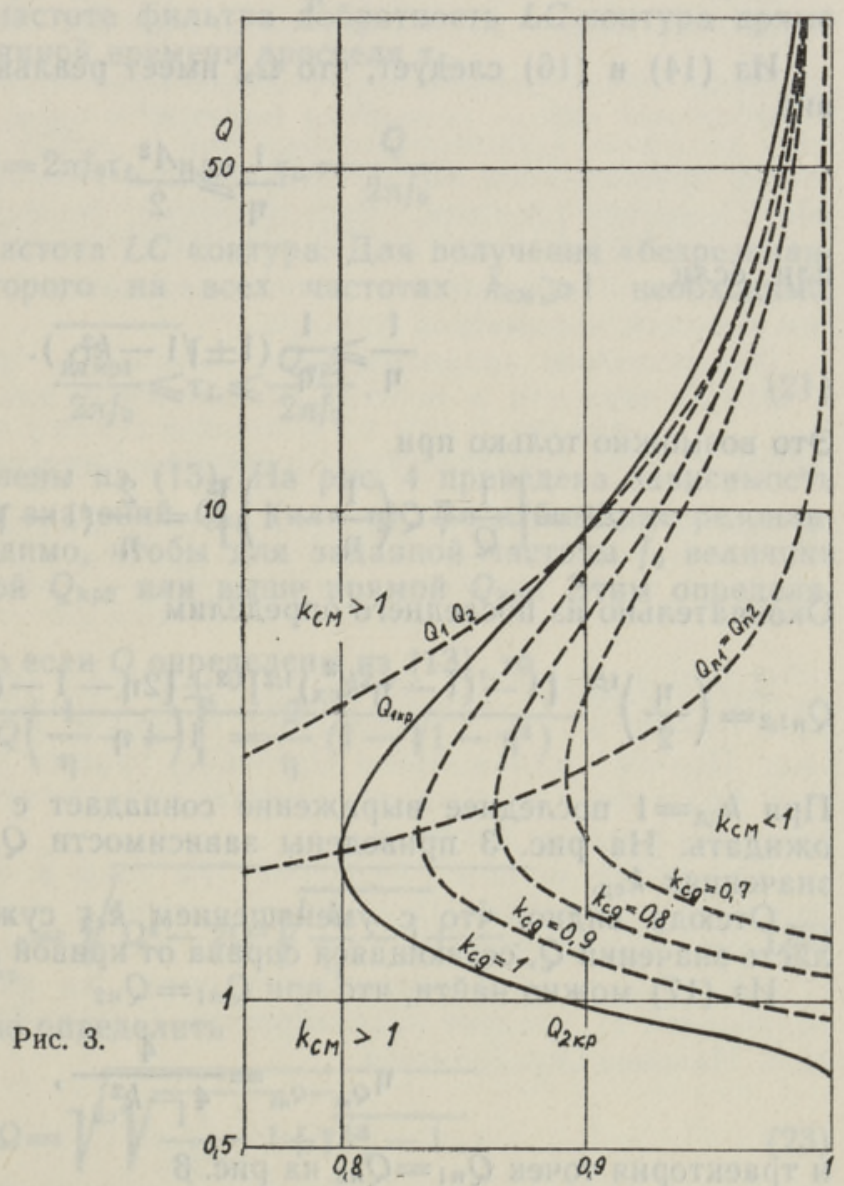


Таким образом, во избежание резонансного увеличения фильтром входного напряжения при любых значениях $\eta$ добротность $L C$-контура должна быть равной или меньше величины $1 / \sqrt{2}$.

Если допустить некоторое увеличение напряжения пульсаций вблизи резонансной частоты, т. е. задать минимальный допустимый $k_{\text {сд }}<1$, можно найти предельные величины $Q_{n 1}<Q_{\mathrm{kp1}}$ и $Q_{\mathrm{n} 2}>Q_{\mathrm{kp} 2}$, подставляя величину $\Omega_{\mathrm{M}}$ из (9) в (5) и приравнять последнюю к задаваемой $k_{\text {сд }}<1$.

Обозначив

$$
\frac{1}{Q}+Q\left(\frac{1}{\eta}-1\right)=A
$$

имеем из (9)

$$
\Omega_{\mathrm{M}}=\sqrt{\frac{1}{\eta}-\frac{1}{2} A^{2}}
$$

и из (5)

$$
k_{\text {сд }}=\sqrt{\left(\frac{1}{\eta}-\Omega_{\mathrm{M}}^{2}\right)^{2}+\Omega_{\mathrm{M}}^{2} A^{2}} .
$$

Из последнего найдем

$$
A^{2}=\frac{2}{\eta}\left(1 \pm \sqrt{1-k_{\mathrm{cd}}^{2}}\right)
$$

Из (14) и (16) следует, что $\Omega_{\mathrm{M}}$ имеет реальные величины при условии

$$
\frac{1}{\eta} \geqslant \frac{A^{2}}{2}
$$

или, если

$$
\frac{1}{\eta} \geqslant \frac{1}{\eta}\left(1 \pm \sqrt{1-k_{\mathrm{cd}}^{2}}\right) .
$$

Это возможно только при

$$
A^{2}=\left[\frac{1}{Q}+Q\left(\frac{1}{\eta}-1\right)\right]^{2}=\frac{2}{\eta}\left(1-\sqrt{1-k_{\mathrm{c \mu}}^{2}}\right) .
$$

Окончательно из последнего определим

$Q_{n 1,2}=\left(\frac{\eta}{2}\right)^{1 / 2} \frac{\left[1-\left(1-\eta^{2} k_{\mathrm{c \mu}}^{2}\right)^{1 / 2}\right]^{1 / 2} \pm\left[2 \eta-1-\left(1-\eta^{2} k_{\mathrm{cR}}^{2}\right)^{1 / 2}\right]^{1 / 2}}{1-\eta}$.

При $k_{\text {сд }}=1$ последнее выражение совпадает с (13), как и следовало ожидать. На рис. 3 приведены зависимости $Q_{n}$ от $\eta$ при различных значениях $k_{\text {сд }}$.

Отсюда видно, что с уменьшением $k_{\text {сд }}$ сужается «запретная» область значений $Q$, остающаяся справа от кривой $k_{\text {сд }}=1$.

Из (17) можно найти, что при $Q_{n 1}=Q_{n 2}$

$$
\eta_{Q_{n 1}=Q_{n 2}}=\frac{4}{4-k_{\text {сд }}^{2}}
$$

и траектория точек $Q_{n 1}=Q_{n 2}$ на рис. 3 


$$
Q_{n 1}=Q_{n 2}=\sqrt{\frac{2}{\eta(1-\eta)^{2}}[1-\sqrt{1-4 \eta(1-\eta)]}}
$$

Таким образом, определяющими параметрами энергетического сглаживающего $L C$-фильтра являются $\eta$ и $Q$. В реальных фильтрах выгодно $\eta$ иметь возможно большим. Повлиять на характеристики фильтра можно путем выбора величин $Q$.

Изменить величину $Q$ можно изменением соотношения $L$ и $C$, изменением соотношения индуктивности и активного сопротивления дросселя (т. е. постоянной времени дросселя), а также использованием активных схем, так как высокую добротность можно достичь введением в цепь дополнительной энергии для компенсации потерь, низкую добротность, наоборот, путем вывода энергии (напр., в виде потерь).

Следует отметить, что вышеприведенные соотношения выведены с учетом активных потерь только в цепи дросселя.

При проектировании фильтра обычно задается минимально необходимый коэффициент фильтрации на заданной частоте. Поэтому выбор величины $Q$ не должен перемещать частотную характеристику фильтра в худшую сторону. Из несколько преобразованного выражения для

$$
Q=\frac{\sqrt{L / C}}{R}=\frac{L}{R} \frac{1}{\sqrt{L C}}=\frac{\tau_{L}}{\sqrt{L C}}
$$

следует, что при постоянной величине произведения $L C$, т. е. при постоянной резонансной частоте фильтра добротность $L C$-контура прямо пропорциональна постоянной времени дросселя $\tau_{L}$.

Из (20) имеем

$$
Q=2 \pi f_{0} \tau_{L} \quad \text { или } \quad \tau_{L}=\frac{Q}{2 \pi f_{0}},
$$

где $f_{0}$ - резонансная частота $L C$ контура. Для получения «безрезонансного» фильтра, у которого на всех частотах $k_{\mathrm{cm}} \geqslant 1$ необходимо, чтобы

$$
\frac{Q_{\mathrm{kp} 1}}{2 \pi f_{0}} \leqslant \tau_{L} \leqslant \frac{Q_{\mathrm{kp} 2}}{2 \pi f_{0}},
$$

где $Q_{\text {kp1 }}$ и $Q_{\text {kp2 }}$ определены из (13). На рис. 4 приведена зависимость $\tau_{L}=f\left(f_{0}\right)$ от различных значений $Q_{\mathrm{kp}}$ (или $\left.\eta\right)$. Во избежание резонансного усиления необходимо, чтобы для заданной частоты $f_{0}$ величина $\tau_{L}$ лежала ниже прямой $Q_{\mathrm{kp2} 2}$ или выше прямой $Q_{\mathrm{kp1}}$. Этим определяется и КПД фильтра.

Можно показать, что если $Q$ определены из (13), то

$$
\left[\frac{1}{Q}+Q\left(\frac{1}{\eta}-1\right)\right]^{2}=\frac{2}{\eta}\left(1-\sqrt{1-\eta^{2}}\right)
$$

и в этом случае

$$
k_{\mathrm{c}=Q_{\mathrm{kp}}}=\sqrt{\Omega^{4}-2 \Omega^{2} \sqrt{\frac{1}{\eta}-1}+\frac{1}{\eta^{2}}} .
$$

Из последнего можно определить

$$
\Omega=\sqrt{\sqrt{\frac{1}{\eta}-1}+\sqrt{k_{\mathrm{c}}^{2}-1}}
$$




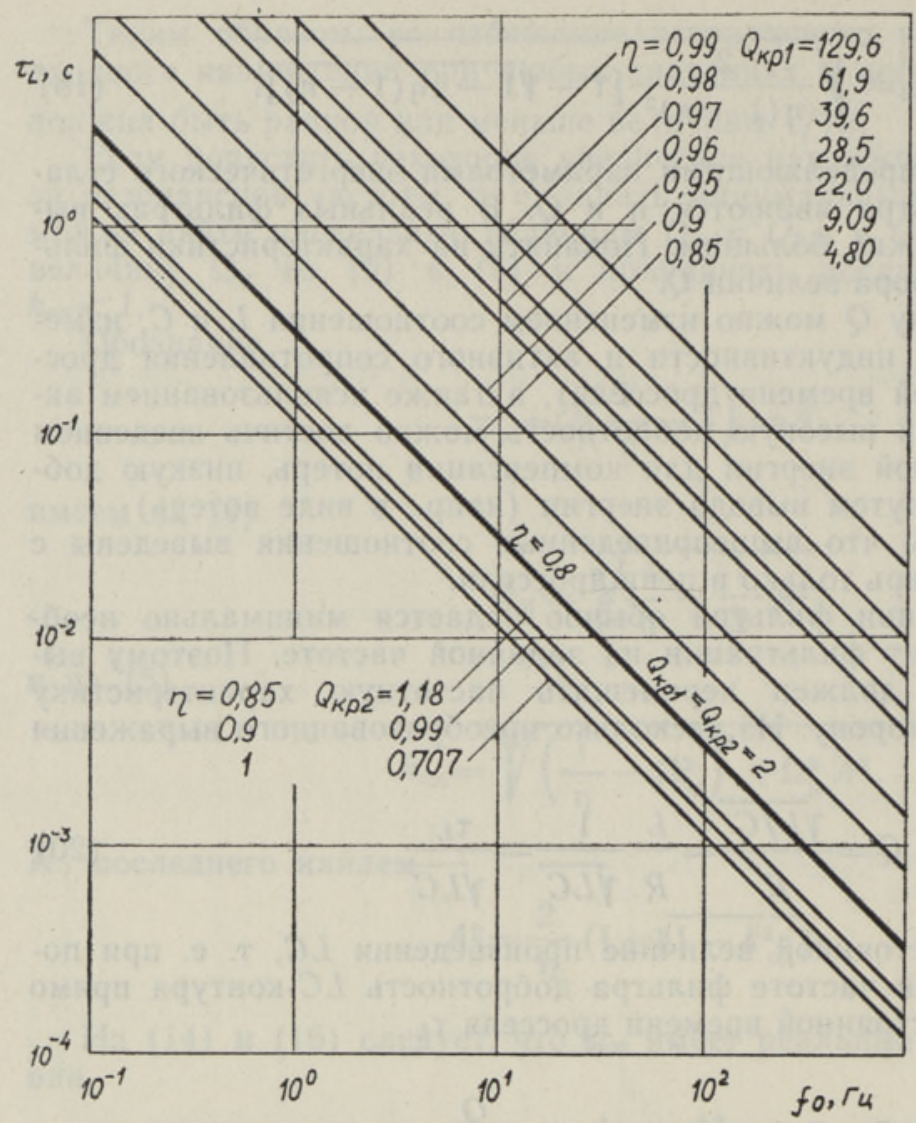

Рнс. 4.

Из приведенного выше следует способ выбора основных параметров элементов сглаживающего фильтра, позволяющего при необходимости избежать (или сократить) резонансного усиления сигнала, а также способ проверки опасности резонанса при выбранных по другим методам параметров фильтра.

Предположим заданным необходимый $k_{\text {c }}$ при частоте $f$. Величину $\eta$ можно задавать в первом приближении или принять ее равной 1 . Затем из (23) определяется $\Omega$, откуда $f_{0}=\frac{f}{\Omega}$. Далее, можно определить произведение

$$
L C=\frac{1}{4 \pi^{2} f_{0}^{2}} .
$$

Потом задавая $Q_{\mathrm{kp1}}$ или $Q_{\mathrm{kp} 2}$, можно из $(21)$ определить $\tau_{L}$. Следует отметить, что $\tau_{L}$ возрастает с ростом массы и габаритов дросселя. Поэтому минимальное $Q_{\mathrm{kp}}=Q_{\mathrm{kp} 2}$ удается задавать при маломощных фильтрах (на токи до десятки ампер), что дает и минимальные габариты и массы дросселя.

Для более мощных фильтров (на токи в сотни и тысячи ампер) приходится задавать $Q_{\mathrm{kp1}}$ предварительно, оценивая величину КПД фильтра. Как следует из рис. 2, при «безрезонансном» фильтре (кривая 3) $Q_{\mathrm{kp1}}$ и $Q_{\mathrm{kp2}}$ различаются почти на два порядка. Исходя из полученной $\tau_{L}$, следует проектировать (или выбрать из каталога) дроссель. Наконец определяется

$$
C=\frac{1}{4 \pi^{2} f_{0} L} .
$$


Повторный расчет по этой же схеме позволяет уточнить и опредёлить окончательные величины электрических параметров основных элементов фильтра.

Если заданы необходимые $k_{\text {c }}$ на нескольких частотах, то в качестве $f_{0}$ для расчета следует принимать наименьшее его значение.

По вышеприведенным формулам и графикам можно также оценить опасность возникновения и величину резонансных явлений у фильтра, параметры элементов которого выбраны исходя из других методик.

\section{ЛИ ТЕ Р А Т У Р А}

1. Тамкиви П. И. В кн.: Уменьшение искажений в цепях с силовыми полупроводниковыми преобразователями. Таллин, АН ЭССР, 1981, 8-10.

2. Walter F. Praeg. IEEE Trans., IECI-17, № 1, 16-22 (1970).

3. Stipaničev, D. Damping of Resonances in REI Filters. Proc. 3rd Symp. on EMC. Rotterdam, $1979,547-552$.

4. Неудорф Р. В кн.: Сглаживающие фильтры. Таллин, АН ЭССР, 1973, $112-118$.

Институт термофизики и электрофизики Академии наук Эстонской ССР
Поступила в редакцию 1/VI 1987

\section{P. TAMKIVI}

\section{T-KUJULISE ENERGEETILISE SILUMISFILTRI OLDISTATUD KARAKTERISTIKUD}

Artiklis on tuletatud aktiivkoormusel töötava $\Gamma$-kujulise silumisfiltri ja faasikarakteristiku avaldused lähtudes kahest kriteriaalsest parameetrist - filtri kasutegurist ja $L C$ kontuuri hüvetegurist. Näidatakse, et filtri sageduskarakteristikud on identsed kahel erineval hüveteguri väärtusel. Esitatakse meetod ja tuletatakse kriteeriumid filtri elementide valikuks, mis võimaldab etteantud tasemeni maha suruda resonantsist tingitud signạali võimenduse efekti ilma spetsiaalseid $R C$ demfeerimisahelaid kasutamata.

\section{P. TAMKIVI}

\section{GENERALIZED CHARACTERISTICS OF THE T-SHAPED POWER-SMOOTHING FILTER}

In the present paper the expressions of the smoothing factor and phase characteristic to $\Gamma$-shaped smoothing filters operating on active load have been derived. They are based on two criterion parameters - filter efficiency and $Q$-factor of the $L C$ circuit. It is shown that the frequency characteristics are identical for two different values of the $Q$-factor.

A method is presented and criterions are derived for the selection of filter units, which enables to suppress the effect of the signal gain due to resonance without applying special $R C$ damping circuits. 